\title{
Qualidade microbiológica e composição de farinhas de resíduos da produção de suco de frutas em diferentes granulometrias
}

\author{
Microbiological quality and composition of flour from fruit juice production \\ residues with different granulometries
}

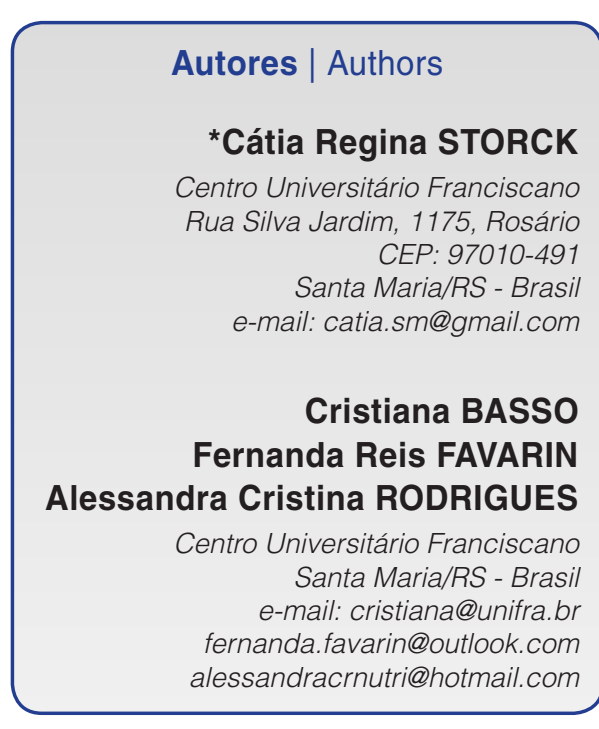

*Autor Correspondente / Corresponding Author

Recebido: Abr. 04, 2015

Aprovado: Jan. 07, 2016

\section{Resumo}

Os resíduos gerados na indústria de sucos têm sido subaproveitados, desperdiçando um produto que pode apresentar potencial para ser incluído na alimentação humana. Assim, o objetivo do estudo foi realizar análise microbiológica e da composição nutricional das farinhas do resíduo de suco de uva, maçã, laranja e acerola, com diferentes granulometrias. Os resíduos da produção de suco foram obtidos através de doação de uma indústria do município de Bento Gonçalves-RS, sendo então secos e moídos para obter a farinha em diferentes granulometrias $(>0,600 \mathrm{~mm} ; 0,600 \mathrm{~mm} ; 0,425 \mathrm{~mm} ; 0,300 \mathrm{~mm} ; \leq 0,250 \mathrm{~mm})$. As amostras de farinha foram submetidas à análise de composição centesimal, acidez, pH, Brix e polifenóis totais. As análises microbiológicas incluíram contagem de coliformes termotolerantes e pesquisa de Salmonella spp. Os resultados indicaram qualidade microbiológica satisfatória. Quanto à composição centesimal, os resultados mostraram que a granulometria influenciou o valor nutricional das farinhas e que os valores energéticos das farinhas do resíduo de acerola, laranja e uva foram inversamente proporcionais ao tamanho da partícula. Todas as farinhas analisadas podem ser consideradas ricas em fibras, cujos valores variaram de $11,2 \%$ a $54,5 \%$. As farinhas de uva e acerola apresentaram maiores teores de proteínas, $8,2 \%$ a $15,6 \%$ e $8,3 \%$ a $12,1 \%$, respectivamente. As farinhas do resíduo de laranja e uva apresentaram elevados teores de polifenóis totais. Portanto, o uso destes resíduos na alimentação humana pode ser recomendado, melhorando o valor nutricional de preparações.

Palavras-chave: Resíduos industriais; Polifenóis; Fibra alimentar; Aproveitamento integral dos alimentos.

\section{Summary}

Residues obtained from the juice industry have been underused, wasting a product that might present potential to be included in food for humans. Thus the objective of the present study was to carry out microbiological and nutritional compositional analyses of the flours made from grape, apple, orange and acerola juice residues, with different granulometries. The juice residues were obtained by donation from an industry in Bento Gonçalves (RS/Brazil), dried and ground in order to obtain flours with different granulometries $(>0.600,0.600 \mathrm{~mm}, 0.425 \mathrm{~mm}$, $0.300 \mathrm{~mm}$ and $\leq 0.250 \mathrm{~mm}$ ). The flour samples were submitted to an analysis of the proximate composition, acidity, $\mathrm{pH},{ }^{\circ} \mathrm{Brix}$ and total polyphenols. The microbiological analysis consisted of enumerating heat tolerant coliforms and testing for the presence of Salmonella spp. The results indicated satisfactory microbiological quality. The proximate composition showed that granulometry influenced the nutritional values of the flours and that the energy values of flours obtained from acerola, orange and grape residues were inversely proportional to the particle size. All the flours analysed could be considered rich in fibres, with values varying from $11.2 \%$ to $54.5 \%$. The grape and acerola flours presented higher protein levels from $8.2 \%$ to $15.6 \%$ and from $8.3 \%$ to $12.1 \%$, respectively - and the orange and grape residue flours presented high levels of total polyphenols. Thus the use of such residues in human nutrition can be recommended, improving the nutritional value of food preparations.

Key words: Industrial residues; Polyphenols; Dietary fiber; Whole food use. 


\section{Introdução}

A fruticultura mundial foi responsável pela produção de 773,8 milhões de toneladas no ano de 2012, sendo o Brasil o terceiro maior produtor mundial de fruta, com uma produção acima de 41 milhões de toneladas (5,3\% do total) no ano de 2012, sendo que a Região Sul colaborou com $7,9 \%$ desta produção. As principais frutas cultivadas foram banana, melancia, maçã, laranja e uva, que, juntas, responderam por $59 \%$ do volume total da fruticultura mundial. No Brasil, a principal fruta cultivada é a laranja, correspondendo a $42 \%$ do volume total (SEAB, 2015).

Além da demanda por frutas frescas, o mercado de sucos e polpas é crescente, principalmente por preservar as características químicas e sensoriais da fruta fresca, e o consumidor poder encontrá-las durante o ano todo, sem ter que se preocupar com a época e o amadurecimento (ABUD; NARAIN, 2009). A produção e a comercialização de sucos de frutas têm crescido nos últimos anos, sendo que, em 2010, foram consumidos 550 milhões de litros de sucos de todos os sabores no Brasil, segundo o Instituto Brasileiro de Frutas (IBRAF, 2011); houve, por isso, uma significativa geração de resíduos.

Entre as frutas utilizadas para produção de suco, a laranja está entre as mais produzidas e consumidas no mundo, sendo que sua produção ultrapassa 68 milhões de toneladas/ano (SEAB, 2015). No Brasil, 80\% da produção resulta em suco industrializado, sendo que $98 \%$ são destinados para exportação, representando $53 \%$ do suco comercializado mundialmente; gera-se, dessa forma, uma grande quantidade de resíduos (NEVES et al., 2010).

Outra fruta utilizada para produção de sucos é a uva e, segundo Mello (2010), a produção de suco de uva integral aumentou 67\% em 2010 em relação ao ano anterior, no Rio Grande do Sul. O bagaço contém compostos que permanecem, mesmo depois da elaboração do suco, como antioxidantes e corantes, e outros compostos com atividades potencialmente funcionais (CAMPOS, 2005), evidenciando o seu alto potencial para elaboração de subprodutos destinados ao consumo humano ou animal.

A cultura da maçã também é uma atividade econômica importante na Região Sul do País, contribuindo com aproximadamente $1,5 \%$ da produção mundial (ABPM, 2013). Em 2012, a produção nacional totalizou 1,22 milhão de toneladas da fruta, sendo que o Rio Grande do Sul foi responsável por aproximadamente $47 \%$ do total (LEVANTAMENTO..., 2013). No Brasil, aproximadamente $15 \%$ da produção nacional de maçã (descarte) é transformada em suco, sendo que a maior parte se destina à exportação.

Pelo seu potencial como fonte natural de vitamina $\mathrm{C}$ e pela sua capacidade de aproveitamento industrial, a aceroleira tem atraído o interesse dos fruticultores e passou a ter importância econômica em várias regiões do Brasil (NOGUEIRA et al., 2002). A acerola também possui um elevado teor de antocianinas e carotenoides (ROSS; KASUM, 2002), pigmentos antioxidantes que, quando combinados, são responsáveis pela coloração vermelha dos frutos (LIMA et al., 2003). Apesar de ser fonte destes constituintes nutricionais, não se acredita no potencial de comercialização da acerola fresca, mas sim no processamento e na conservação de sua polpa, e na produção do seu suco, pois a qualidade da fruta diminui rapidamente após a colheita. Desta forma, a geração de resíduos, a partir da produção do suco ou polpa, é aumentada.

Os resíduos da indústria de sucos são geralmente utilizados para alimentação animal; no entanto, alguns estudos têm sido realizados para avaliar a qualidade e o potencial dos resíduos da produção de suco para utilização na alimentação humana (ABUD; NARAIN, 2009; AQUINO et al., 2010; COELHO; WOSIACKI, 2010; NATIVIDADE, 2010; CÓRDOVA et al., 2013). Entretanto, a maioria destes estudos apenas realiza a quantificação de cada fração, não abordando as diferenças na composição das farinhas com diferentes granulometrias, o que pode auxiliar para diferentes usos na alimentação humana. Em estudo realizado por Coelho e Wosiacki (2010), com farinha de bagaço de maçã, foi verificado que $59,3 \%$ da farinha apresentou granulometria inferior a $0,5 \mathrm{~mm}$, e $30,7 \%$ apresentaram granulometria de $0,5 \mathrm{~mm}$; observe-se que não foi verificada a composição nutricional de cada fração.

Uma das características nutricionais importantes das frutas é seu conteúdo de compostos antioxidantes, como os polifenóis, os quais podem estar presentes também nos resíduos das mesmas. Os compostos fenólicos são denominados antioxidantes primários, porque agem como sequestradores de radicais livres e bloqueadores de reações em cadeia (MOREIRA; MANCINI-FILHO, 2004).

Diante do exposto, o presente trabalho teve como objetivo realizar a análise microbiológica e da composição nutricional das farinhas do resíduo de suco de uva, maçã, laranja e acerola, com diferentes granulometrias.

\section{Material e métodos}

\subsection{Amostra}

Os resíduos da produção de suco de acerola, laranja, maçã e uva foram obtidos por meio da doação de uma indústria no município de Bento Gonçalves-RS. Os resíduos foram retirados de dentro da fábrica após serem pasteurizados e foram secos em estufa com circulação forçada de ar (De Leo), mantendo a temperatura de 50 a $55^{\circ} \mathrm{C}$ até peso constante. Após a 
secagem, o resíduo foi moído em macromoinho de facas tipo Willey TE-650 (Tecnal), com apenas uma passagem, para obter uma farinha. A farinha foi peneirada em peneiras de diferentes granulometrias: 0,600 mm; 0,425 mm; 0,300 mm; 0,250 mm. A farinha que não passou na peneira de $0,600 \mathrm{~mm}$ foi nomeada com granulometria $>0,600 \mathrm{~mm}$ e as que passaram pela peneira de 0,250 $\mathrm{mm}$ foram nomeadas com granulometria $\leq 0,250 \mathrm{~mm}$. Em seguida, as quantidades retidas em cada peneira foram pesadas e expressas em porcentagem. As farinhas foram acondicionadas em potes plásticos, sob o abrigo da luz e em lugar fresco, até o momento das análises. Todos os equipamentos e utensílios utilizados foram previamente higienizados com álcool 70\% para não haver contaminação das amostras.

\subsection{Análises microbiológicas}

As amostras foram submetidas às análises microbiológicas de determinação do Número Mais Provável por grama de amostra (NMP/g) para coliformes termotolerantes e pesquisa de Salmonella spp., com presença ou ausência, em $25 \mathrm{~g}$ de amostra (WANDERZANT; SPLITTSTOESSER, 1992). Os resultados das análises microbiológicas foram comparados aos padrões estabelecidos pela RDC n. ${ }^{\circ} 12$ de 02/01/2001 do Ministério da Saúde (BRASIL, 2001), a qual aprova, no Art. $1^{\circ}$, o Regulamento Técnico sobre padrões microbiológicos para alimentos, e comparados também com publicações científicas.

\subsection{Análises bromatológicas}

A composição centesimal foi realizada de acordo com a Horwitz (2002). O teor de proteína bruta foi quantificado pelo método de Kjeldahl e o de lipídeos totais foi determinado pelo método de Soxhlet, usando éter de petróleo. A umidade foi determinada em estufa a $105^{\circ} \mathrm{C}$ até a obtenção de peso constante e as cinzas, em mufla a $550^{\circ} \mathrm{C}$. A quantificação de fibra bruta foi determinada pelo método da fibra bruta (BRASIL, 1991), apesar de não ser o método mais indicado, pois superestima os valores de carboidratos e valor energético (MONRO; BURLINGAME, 1996; Ll et al., 2002). Carboidratos digeríveis foram estimados por diferença, subtraindo-se de $100 \%$ a soma dos valores obtidos para as determinações anteriores. Os resultados foram expressos em base úmida. O valor energético foi calculado utilizando-se os seguintes fatores de conversão de Atwater: carboidratos, $4 \mathrm{kcal} \mathrm{g}^{-1}$; proteínas, 4 kcal g ${ }^{-1}$; lipídios, 9 kcal g-1 (MENDEZ et al., 1995).

Para a análise de polifenóis, estes foram extraídos utilizando-se solução de metanol 50\% e acetona 70\%. A concentração de polifenóis totais foi determinada pelo método colorimétrico descrito por Singleton e Rossi (1965), empregando uma curva padrão de ácido gálico.

A determinação da acidez foi baseada na titulação de neutralização de ácidos com solução padronizada de álcali (solução de $\mathrm{NaOH} \mathrm{0,1} \mathrm{N)} \mathrm{e} \mathrm{com} \mathrm{o} \mathrm{uso} \mathrm{de} \mathrm{indicador}$ fenolftaleína (IAL, 1985). O ácido orgânico utilizado foi o ácido cítrico, com peso molecular de $192 \mathrm{~g} \mathrm{~mol}^{-1}$ e 2 hidrogênios ionizáveis. $\mathrm{O}$ pH foi determinado em pHmetro (Digimed) e o ${ }^{\circ}$ Brix em refratômetro (Biobrix). Estas análises foram realizadas apenas nas amostras de maior e menor granulometrias.

\subsection{Análise estatística}

Para avaliação estatística, foi realizada análise de variância (ANOVA) e as médias dos parâmetros analisados para cada farinha nas diferentes granulometrias, para cada fruta em separado, foram comparadas pelo teste de Duncan $(p<0,05)$, utilizando-se o programa estatístico Statistical Package for the Social Sciences 14.0.

\section{Resultados e discussão}

\subsection{Análise microbiológica}

Os resultados das análises microbiológicas das farinhas de resíduos da produção de suco de frutas (Tabela 1) indicam que todas as farinhas analisadas apresentaram qualidade microbiológica satisfatória, estando aptas a serem utilizadas na alimentação humana, já que atendem à RDC n. ${ }^{\circ} 12$ de janeiro de 2001 (BRASIL, 2001). Não foi encontrada Salmonella spp. em nenhuma das amostras. Esses resultados favoráveis possivelmente se devem ao fato de os resíduos terem sido retirados da indústria já pasteurizados e também pelos programas de qualidade empregados no local, como ISO 9001:2008; HACCP; FSSC 22.000; GMA; BPF e BPA. Conforme Natividade (2010), a adoção de boas práticas de fabricação durante a manipulação de alimentos é um fator imprescindível na determinação de seu padrão sanitário, assegurando assim a inocuidade do produto final.

Outro fator também a ser considerado é em relação ao pH, já que, segundo Germano (2001), normalmente as bactérias conseguem se desenvolver em uma faixa de $\mathrm{pH}$ que varia entre 5 e 8 , sendo que, nesta pesquisa,

Tabela 1. Resultados das análises microbiológicas das farinhas de resíduo da produção de suco de acerola, laranja, maçã e uva.

\begin{tabular}{lccccc}
\multicolumn{1}{c}{ Grupo microbiano } & Acerola & Laranja & Maçã & Uva & Padrões microbiológicos* $^{*}$ \\
Coliformes termotolerantes NMP.g ${ }^{-1}$ & $<3$ & $<3$ & $<3$ & $<3$ & $10^{2} \mathrm{~g}^{-1}\left(\mathrm{máx}^{\prime}\right)$ \\
Salmonella sp. $25 \mathrm{~g}^{-1}$ & Ausente & Ausente & Ausente & Ausente & Ausência em $25 \mathrm{~g}$ \\
\hline
\end{tabular}

*Fonte: Brasil (2001), para grupo de alimentos Frutas, Produtos de Frutas e Similares. 
o maior valor de $\mathrm{pH}$ encontrado foi de 4,2. Também os compostos fenólicos presentes agem como agentes antimicrobianos naturalmente presentes nessas farinhas (PUUPPONEN-PIMIA et al., 2001).

Abud e Narain (2009) analisaram os resíduos do processamento de polpa de acerola, umbu, goiaba e maracujá, e verificaram que as farinhas obtidas possuíam características microbiológicas aceitáveis para o consumo humano. Da mesma forma, Coelho e Wosiacki (2010) verificaram que a farinha de bagaço de maçã apresentou características microbiológicas adequadas, com valores abaixo dos limites aceitos pela legislação brasileira.

\subsection{Composição Centesimal}

Os resultados da análise granulométrica das farinhas dos resíduos da produção de suco de frutas estão apresentados na Tabela 2. De acordo com a análise granulométrica, a farinha de resíduo da produção de suco de acerola (FRA) apresentou maior porcentagem na granulometria $\leq 0,250 \mathrm{~mm}$ do que as outras farinhas, enquanto que, nas farinhas de resíduo da produção de suco de laranja (FRL), maçã (FRM) e uva (FRU) foi constatada uma maior porcentagem na granulometria $>0,600 \mathrm{~mm}$. De acordo com Borges et al. (2003), o tamanho de partícula influencia diretamente a capacidade de absorção de água, o tempo de mistura e as características sensoriais, como aparência, sabor e textura. A influência da granulometria de farinhas nas propriedades de produtos de panificação ainda não foi totalmente elucidada, embora seja aceito que partículas finas e uniformes promovem maior dispersibilidade da farinha na massa (BORGES et al., 2011). De acordo com Matias et al. (2005), em estudo com bagaços de goiaba e caju, as partículas retidas das peneiras entre 0,230 mm e 0,149 mm foram consideradas as mais adequadas para serem incorporadas ao alimento.

Os resultados da composição centesimal e de polifenóis das farinhas dos resíduos da produção de suco de frutas são apresentados na Tabela 3. Pode observar que os valores energéticos de FRA, FRL e FRU foram inversamente proporcionais ao tamanho da partícula, sendo que a FRM foi a única em que se verificou um decréscimo com a granulometria. Estes valores estão ligados principalmente à quantidade de carboidratos e lipídios encontrados nas amostras. Aguiar et al. (2010) pesquisaram a farinha das sementes de acerola e encontraram valor energético maior (366 kcal $100 \mathrm{~g}^{-1} \mathrm{~b} . \mathrm{s}$.) do que o verificado para todas granulometrias.

Em relação à quantidade de carboidratos, a granulometria também influenciou os teores, sendo que a FRU apresentou os menores índices de carboidratos $(30,5 \%$ a $45,8 \%)$ e a FRM apresentou os maiores $(67,4 \%$ a $74,9 \%$ ). O critério para a adição destas farinhas em alimentos pode ser o objetivo nutricional, uma vez que estas podem ser utilizadas em substituição à farinha de trigo, que contém $75,1 \%$ de carboidratos (NEPA; UNICAMP, 2006). Aguiar et al. (2010) verificaram valor superior para a farinha de semente de acerola $(63,2 \%)$.

Apesar do tipo de análise realizada (fibra bruta), a qual pode subestimar os teores de fibra, todas as farinhas analisadas podem ser consideradas ricas em fibra, pois, de acordo com a legislação brasileira, estas apresentam um teor superior a $6 \%$ de fibras (BRASIL, 2012). Os teores de fibra bruta foram maiores em FRA (33,2\% a 43,1\%) e FRU (21,3\% a 54,5\%), sendo que, com a diminuição da granulometria, houve diminuição significativa do teor de fibra. A FRM, quando comparada com a farinha do bagaço da maçã (46,3\% b.s.), pesquisada por Coelho e Wosiacki (2010), possuiu um valor menor; isso pode ser explicado pelo método utilizado, pois, na técnica de fibra bruta, ocorre perda de fibra solúvel da amostra e, como a maçã é rica em pectina, uma fibra solúvel, ela vai apresentar um menor teor de fibra. Portanto, a adição das farinhas de resíduo da produção de suco em produtos alimentícios pode agregar propriedades funcionais interessantes para a saúde humana, enriquecendo tais produtos.

O comportamento das farinhas em relação ao teor de proteína foi diferente dos verificados nas fibras, em que se observou que conforme diminuiu a granulometria, aumentou significativamente o teor deste nutriente. As farinhas de uva $(8,2 \%$ a $15,6 \%)$ e acerola $(8,3 \%$ a $12,1 \%$ ) foram as que apresentaram maiores teores. A FRM, quando comparada com a farinha do bagaço da maçã pesquisado por Coelho e Wosiacki (2010), apresentou valor semelhante $(3,61 \%$ b.s.) e a FRA apresentou valor menor quando comparado com a farinha da semente de acerola (18,7\%b.s.) pesquisada por Aguiar et al. (2010). Ao comparar com o teor de proteína da farinha de trigo $(9,8 \%)$ (NEPA; UNICAMP, 2006), percebemos que as

Tabela 2. Distribuição do tamanho de partículas das farinhas de resíduos da produção de suco de acerola, laranja, maçã e uva.

\begin{tabular}{|c|c|c|c|c|}
\hline Granulometria (mm) & Acerola (\%) & Laranja (\%) & Maçã (\%) & Uva (\%) \\
\hline$>0,600$ & 12 & 52 & 37 & 46 \\
\hline 0,600 & 15 & 18 & 18 & 21 \\
\hline 0,425 & 26 & 11 & 13 & 9 \\
\hline 0,300 & 10 & 8 & 8 & 8 \\
\hline$\leq 0,250$ & 37 & 12 & 24 & 17 \\
\hline
\end{tabular}


Tabela 3. Composição centesimal e polifenóis de farinhas dos resíduos da produção de suco de acerola, laranja, maçã e uva, em diferentes granulometrias (em $100 \mathrm{~g}$ b.s.).

\begin{tabular}{|c|c|c|c|c|c|c|c|}
\hline $\begin{array}{c}\text { Granulometria } \\
(\mathrm{mm})\end{array}$ & $\begin{array}{c}\text { Valor } \\
\text { energético } \\
\text { (kcal) }\end{array}$ & $\begin{array}{l}\text { CHO } \\
\text { (g) }\end{array}$ & $\begin{array}{l}\text { FB } \\
\text { (g) }\end{array}$ & $\begin{array}{l}\text { PTN } \\
\text { (g) }\end{array}$ & $\begin{array}{l}\text { ㄴIP } \\
\text { (g) }\end{array}$ & $\begin{array}{l}C Z \\
\text { (g) }\end{array}$ & $\begin{array}{l}\text { Polifenóis } \\
\text { (mg) }\end{array}$ \\
\hline \multicolumn{8}{|c|}{ Acerola } \\
\hline$>0,600$ & 225,6 & 45,3 & $43,1^{a} \pm 1,2$ & $8,3^{e} \pm 0,0$ & $1,24^{d} \pm 0,07$ & $2,03^{b} \pm 0,03$ & $578^{a} \pm 69$ \\
\hline 0,600 & 239,4 & 46,8 & $40,4^{b} \pm 0,3$ & $9,2^{\mathrm{d}} \pm 0,1$ & $1,71^{\mathrm{c}} \pm 0,16$ & $1,93^{b} \pm 0,10$ & - \\
\hline 0,425 & 250,5 & 47,8 & $37,9^{b} \pm 1,2$ & $10,3^{c} \pm 0,3$ & $1,99^{b c} \pm 0,18$ & $1,95^{b} \pm 0,02$ & - \\
\hline 0,300 & 263,1 & 49,8 & $35,0^{c} \pm 0,9$ & $11,1^{\mathrm{b}} \pm 0,2$ & $2,19^{b} \pm 0,01$ & $1,95^{b} \pm 0,02$ & - \\
\hline$\leq 0,250$ & 272,7 & 49,1 & $33,2^{\mathrm{c}} \pm 0,8$ & $12,1^{\mathrm{a}} \pm 0,3$ & $3,10^{a} \pm 0,13$ & $2,51^{a} \pm 0,11$ & $222^{b} \pm 41$ \\
\hline \multicolumn{8}{|c|}{ Laranja } \\
\hline$>0,600$ & 341,3 & 73,4 & $14,4^{b} \pm 0,3$ & $6,20^{\circ} \pm 0,18$ & $2,53^{\mathrm{e}} \pm 0,26$ & $3,40^{b} \pm 0,12$ & $1156^{b} \pm 29$ \\
\hline 0,600 & 339,7 & 69,0 & $16,8^{a} \pm 0,6$ & $6,50^{\circ} \pm 0,29$ & $4,19^{d} \pm 0,05$ & $3,47^{b} \pm 0,08$ & - \\
\hline 0,425 & 346,9 & 66,6 & $16,6^{\mathrm{a}} \pm 0,8$ & $8,15^{b} \pm 0,47$ & $5,34^{c} \pm 0,45$ & $3,39^{b} \pm 0,15$ & - \\
\hline 0,300 & 353,0 & 63,4 & $16,7^{a} \pm 0,5$ & $9,38^{a} \pm 0,31$ & $6,86^{b} \pm 0,17$ & $3,62^{\mathrm{ab}} \pm 0,14$ & - \\
\hline$\leq 0,250$ & 386,7 & 65,8 & $11,2^{\mathrm{c}} \pm 0,3$ & $9,87^{a} \pm 0,38$ & $9,35^{\mathrm{a}} \pm 0,57$ & $3,83^{a} \pm 0,06$ & $2352^{a} \pm 49$ \\
\hline \multicolumn{8}{|c|}{ Maçã } \\
\hline$>0,600$ & 318,1 & 74,9 & $17,2^{\mathrm{b}} \pm 0,4$ & $2,7^{b} \pm 0,0$ & $0,87^{d} \pm 0,03$ & $4,38^{d} \pm 0,25$ & $329^{a} \pm 19$ \\
\hline 0,600 & 328,3 & 74,9 & $15,4^{\mathrm{c}} \pm 0,4$ & $3,2^{a} \pm 0,1$ & $1,75^{c} \pm 0,12$ & $4,68^{d} \pm 0,27$ & - \\
\hline 0,425 & 307,8 & 69,1 & $19,7^{\mathrm{a}} \pm 0,1$ & $3,5^{a} \pm 0,3$ & $1,93^{b c} \pm 0,07$ & $5,79^{c} \pm 0,40$ & - \\
\hline 0,300 & 322,8 & 70,5 & $15,4^{\mathrm{c}} \pm 0,0$ & $3,4^{a} \pm 0,1$ & $3,02^{\mathrm{a}} \pm 0,12$ & $7,72^{b} \pm 0,44$ & - \\
\hline$\leq 0,250$ & 298,6 & 67,4 & $11,3^{\mathrm{d}} \pm 0,4$ & $3,2^{a} \pm 0,0$ & $2,28^{b} \pm 0,32$ & $16,86^{a} \pm 0,33$ & $309^{a} \pm 56$ \\
\hline \multicolumn{8}{|c|}{ Uva } \\
\hline$>0,600$ & 205,2 & 30,5 & $54,5^{\mathrm{a}} \pm 1,4$ & $8,2^{d} \pm 0,1$ & $5,6^{d} \pm 0,1$ & $1,23^{b} \pm 0,61$ & $1718^{b} \pm 180$ \\
\hline 0,600 & 286,3 & 38,5 & $38,1^{b} \pm 1,3$ & $11,6^{\mathrm{c}} \pm 0,4$ & $9,6^{c} \pm 0,2$ & $2,28^{a} \pm 0,20$ & - \\
\hline 0,425 & 346,2 & 41,3 & $28,1^{c} \pm 0,9$ & $14,1^{b} \pm 0,3$ & $13,9^{b} \pm 0,3$ & $2,61^{a} \pm 0,37$ & - \\
\hline 0,300 & 353,9 & 45,8 & $24,2^{\mathrm{cd}} \pm 1,6$ & $14,6^{\mathrm{ab}} \pm 0,4$ & $12,5^{\mathrm{b}} \pm 0,9$ & $2,91^{a} \pm 0,05$ & - \\
\hline$\leq 0,250$ & 389,7 & 42,9 & $21,3^{d} \pm 1,8$ & $15,6^{a} \pm 0,7$ & $17,3^{a} \pm 0,6$ & $2,86^{a} \pm 0,06$ & $3805^{a} \pm 250$ \\
\hline
\end{tabular}

b.s. = base seca; $\mathrm{CHO}=$ carboidrato; FB = fibra bruta; PTN = proteína; LIP = lipídio; CZ = cinzas. Médias seguidas de letras iguais na coluna, entre cada resíduo, não diferem significativamente $(p<0,05)$ pelo teste de Duncan.

farinhas de resíduos de frutas são ótimas fontes deste nutriente e que, mesmo as frutas não sendo fontes de proteína, apresentaram quantidades importantes e podem ser adicionadas a produtos com a finalidade de melhorar o teor proteico, principalmente em populações carentes, com menos acesso a alimentos ricos neste nutriente.

Os teores de lipídios das farinhas variaram significativamente entre os tipos de farinhas e entre as granulometrias. Para todas as farinhas, quanto menor a granulometria, maior o teor de lipídios. Os maiores índices de lipídios foram os encontrados em FRU, os quais variaram de $17,3 \%(\leq 0,250 \mathrm{~mm})$ a $5,6 \%(>0,600 \mathrm{~mm})$ e os menores índices foram encontrados em FRA e FRM, que obtiveram variação de $1,24 \%$ a 3,10\% e 0,87\% a 3,02\%, respectivamente.

O teor de cinzas, que remete ao teor de minerais totais de um alimento, também foi influenciado pela granulometria das farinhas, mas em menor significância. O resíduo que apresentou maiores teores de minerais foi o da maçã $(4,38 \%$ a $16,86 \%)$. No entanto, todas as farinhas apresentam quantidades importantes de minerais e, se adicionadas a produtos, podem melhorar o valor nutricional dos mesmos. Aquino et al. (2010) verificaram teores de 3,3\% b.s. de cinzas na farinha de bagaço de acerola, valor inferior à esta pesquisa, enquanto Aguiar et al. (2010) encontraram apenas 0,48\% b.s. de cinzas na farinha de semente de acerola.

As farinhas do resíduo de laranja e uva apresentaram altos teores de polifenóis totais, demonstrando que podem ser indicadas para uso na alimentação humana, proporcionando efeito antioxidante. Em relação ao teor de polifenóis, a FRM foi a única que não apresentou diferença estatística entre as granulometrias (média de $282 \mathrm{mg} 100 \mathrm{~g}^{-1}$ ). A FRU de menor granulometria foi a que apresentou o maior teor de polifenóis (3.568 mg $\left.100 \mathrm{~g}^{-1}\right)$, seguida pela FRL de menor granulometria (2.115 mg $\left.100 \mathrm{~g}^{-1}\right)$. Souza et al. (2011) analisaram resíduo

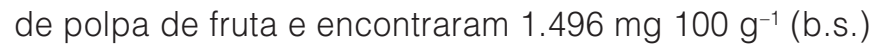
de fenóis totais no resíduo de acerola, valor superior ao deste estudo. Os teores de polifenóis encontrados foram superiores aos verificados por Kuskoski et al. (2006) em polpa de maracujá (20,0 mg $\left.100 \mathrm{~g}^{-1}\right)$, 
Qualidade microbiológica e composição de farinhas de resíduos da produção de suco de frutas em diferentes granulometrias STORCK, C. R. et al.

Tabela 4. Acidez, pH e ${ }^{\circ}$ Brix das farinhas dos resíduos da produção de suco de acerola, laranja, maçã e uva, em diferentes granulometrias.

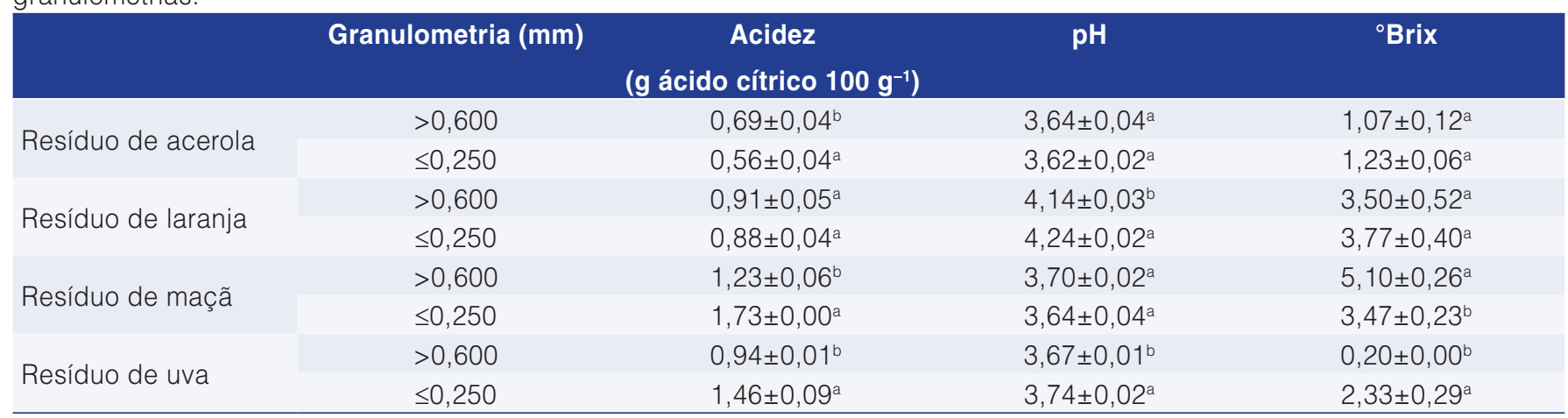

Médias seguidas de letras iguais na linha, entre cada resíduo, não diferem significativamente $(p<0,05)$ pelo teste de Duncan.

goiaba (83,0 mg $\left.100 \mathrm{~g}^{-1}\right)$, abacaxi $\left(21,7 \mathrm{mg} 100 \mathrm{~g}^{-1}\right)$, manga (544, mg $100 \mathrm{~g}^{-1}$ ) e acerola (580,1 mg $100 \mathrm{~g}^{-1}$ ), demonstrando o potencial destas farinhas para uso como antioxidante na elaboração de produtos.

Os resultados das análises de acidez, $\mathrm{pH}$ e ${ }^{\circ}$ Brix das farinhas dos resíduos da produção de suco das frutas encontram-se na Tabela 4, na qual podemos observar que a FRA demonstrou maior acidez $(0,56 \%)$ e a FRM possui o menor teor de acidez (1,73\%). A FRL foi à única amostra que não demonstrou diferença estatística entre as granulometrias.

Em relação ao $\mathrm{pH}$, as farinhas FRA e FRM não apresentaram diferença estatística. $\mathrm{O}$ menor $\mathrm{pH}$ apresentado foi da FRA, que variou entre 3,62 e 3,64, e o maior foi da FRL, que variou entre 4,14 e 4,24. As análises de sólidos solúveis totais das farinhas indicaram diferença estatística em FRM e FRU; já para FRA e FRL não se demonstrou diferença estatística. A FRA apresentou um valor inferior (média de $1,15^{\circ} \mathrm{Brix}$ ), quando comparado com o da farinha do resíduo da acerola, que foi de 47,5Brix em $100 \mathrm{~g}$ (AQUINO et al., 2010); tal fato é, provavelmente, devido às diferenças no processamento e no beneficiamento do produto.

\section{Conclusão}

As farinhas de resíduo da produção de suco podem ser usadas na alimentação humana para enriquecer ou suplementar as preparações alimentícias, pois apresentaram qualidade microbiológica satisfatória e, em sua composição, verificaram-se elevados teores de fibra, proteína, cinzas e polifenóis. As diferentes granulometrias apresentaram diferenças na composição, as quais podem ser consideradas para uso em diferentes produtos alimentícios e com diferentes objetivos nutricionais.

\section{Referências}

ABUD, A. K. S.; NARAIN, N. Incorporação da farinha de resíduo do processamento de polpa de fruta em biscoitos: uma alternativa de combate ao desperdício. Brazilian Journal of Food Technology, Campinas, v. 12, n. 4, p. 257-65, 2009. Disponivel em: <http://bjtt.ital.sp.gov.br/artigos/html/busca/PDF/ v12n4389a.pdf>. Acesso em: 22 set. 2011.

AGUIAR, T. M.; RODRIGUES, F. S.; SANTOS, E. R.; SABAASRUR, A. U. O. Caracterização química e avaliação do valor nutritivo de sementes de acerola. Nutrire: Revista da Sociedade Brasileira de Alimentação e Nutrição, São Paulo, v. 35, n. 2, p. 91-102, 2010. Disponível em: <http://www.revistanutrire.org. br/articles/view/id/4f9b03cc1ef1fac238000008>. Acesso em: 02 maio 2013.

AQUINO, A. C. M. S.; MÓES, R. S.; LEÃO, K. M. M.; FIGUEIREDO, A. V. D.; CASTRO, A. A. Avaliação físico-química e aceitação sensorial de biscoitos tipo cookies elaborados com farinha do resíduo de acerola. Revista do Instituto Adolfo Lutz, São Paulo, v. 69, n. 3, p. 379-86, 2010. Disponível em: <http:// revistas.bvs-vet.org.br/rialutz/article/view/6341/6035>. Acesso em: 02 maio 2013.

ASSOCIAÇÃO BRASILEIRA DE PRODUTORES DE MAÇÃ ABPM. O site da maça brasileira. 2013. Disponível em: <http:// www.abpm.org.br>. Acesso em: 12 jan. 2013.

BORGES, J. T. S.; ASCHERI; J. L. R.; ASCHERI, D. R.; NASCIMENTO, R. E.; FREITAS, A. S. Propriedades de cozimento e caracterização físico-química de macarrão pré-cozido à base de farinha integral de quinoa (Chenopodium quinoa, Wild) e de farinha de arroz (Oryza sativa, L) polido por extrusão termoplástica. Boletim do CEPPA, Curitiba, v. 21, n. 2, p. 303-322, 2003. Disponível em: <http://ojs.c3sl.ufpr.br/ojs/index. php/alimentos/article/view/1167>. Acesso em: 24 ago. 2015.

BORGES, J. T. S.; PIROZI, M. R.; CHAVES, J. B. P.; GERMANI, R.; PAULA, C. D. Caracerização físico-química e reológica das farinhas mistas de trigo e linhaça. Boletim do CEPPA, Curitiba, v. 29, n. 2, p. 159-172, 2011. Disponível em: <http://ojs.c3sl. ufpr.br/ojs/index.php/alimentos/article/view/25478>. Acesso em: 02 abr. 2014.

BRASIL. Agência Nacional de Vigilância Sanitária. Resolução $n^{\circ} 12$, de 2 de janeiro de 2001. Regulamento técnico sobre os padrões microbiológicos para alimentos. Diário Oficial [da] República Federativa do Brasil, Brasília, DF, 12 jan. 2001. 
Qualidade microbiológica e composição de farinhas de resíduos da produção de suco de frutas em diferentes granulometrias STORCK, C. R. et al.

BRASIL. Agência Nacional de Vigilância Sanitária. Resolução, RDC $n^{\circ}$ 54, de 12 de novembro de 2012. Dispõe sobre o Regulamento Técnico sobre Informação Nutricional Complementar. Diário Oficial [da] República Federativa do Brasil, Brasília, DF, 12 nov. 2012.

BRASIL. Portaria 108 de 04 de setembro de 1991. Normas gerais de amostragem para análise de rotina. Método número 11 - Fibra Bruta. Diário Oficial [da] República Federativa do Brasil, Brasília, DF, 17 set. 1991. seção 1; p. 19813.

CAMPOS, L. M. A. S. Obtenção de extratos de bagaço de uva Cabernet Sauvignon (Vitisviníífera): parâmetros de processo e modelagem matemática. 2005. 123 f. Dissertação (Mestrado em Engenharia de Alimentos)-Universidade Federal de Santa Catarina, Florianópolis, 2005.

COELHO, L. M.; WOSIACKI, G. Avaliação sensorial de produtos panificados com adição de farinha de bagaço de maçã. Ciência e Tecnologia de Alimentos, Campinas, v. 30, n. 3, p. 582-588, 2010. Disponível em: <http://www.scielo.br/pdf/cta/v30n3/ v30n3a03.pdf>. Acesso em: 02 maio 2013.

CÓRDOVA, K. V.; GAMA, T. M. M. T. B.; WINTER, C. M. G.; KASKANTZIS NETO, G.; FREITAS, R. J. S. Características físicoquímicas da casca do maracujá amarelo (Passiflora edulis Flavicarpa Degener) obtida por secagem. Boletim do Centro de Pesquisa e Processamento de Alimentos, Curitiba v. 23, n. 2, p. 221-230, 2013. Disponível em: <http://ojs.c3sl.ufpr.br/ojs-2.2.4/index.php/alimentos/ article/view/4491/3497>. Acesso em: 02 maio 2013.

GERMANO, P. M. L. Higiene e vigilância sanitária de alimentos. 2. ed. São Paulo: Varela, 2001. 655 p.

HORWITZ, W. (Ed.). Official methods of analysis of the Association of Official Analytical Chemists. 17th ed. Gaithrsburg: AOAC, 2002. v. 2.

INSTITUTO ADOLFO LUTZ - IAL. Métodos químicos para análise de alimentos. 3. ed. São Paulo: Instituto Adolfo Lutz; 1985.

INSTITUTO BRASILEIRO DE FRUTAS - IBRAF. Contem informações institucionais, técnicas, notícias, projetos, publicações e serviços. São Paulo: IBRAF, 2011. Disponível em: <http://www.ibraf.org.br/>. Acesso em: 31 ago. 2011.

KUSKOSKI, E. M.; ASUERO, A. G.; MORALES, M. T.; FETT, R. Frutos tropicais silvestres e polpas congeladas: atividade antioxidante, polifenóis e antocianinas. Ciência Rural, Santa Maria, v. 36, n. 4, p. 1283-1287, 2006. Disponível em <http://www.scielo.br/scielo. php?pid=s0103-84782006000400037\&script=sci_arttext $>$. Acesso em: 09 dez. 2013.

LEVANTAMENTO SISTEMÁTICO DA PRODUÇÃO AGRÍCOLA. Rio de Janeiro: IBGE, v. 26, n. 7, 2013. 122 p. Disponível em: <http://www.ibge.gov.br/home/estatistica/indicadores/ agropecuaria/lspa/lspa_201307.pdf>. Acesso em: 03 set. 2013.

LI, B. W.; ANDREWS, K. W.; PEHRSSON, P. R. Individual sugars, soluble, and insoluble dietary fiber contents of 70 high consumption foods. Journal of Food Composition and Analisys, v. 15, n. 6, p. 715-723, 2002. http://dx.doi.org/10.1006/ jfca.2002.1096.

LIMA, V. L. A. G.; MÉLO, E. A.; MACIEL, M. I. S.; LIMA, D. E. S. Avaliação do teor de antocianinas em polpa de acerola congelada proveniente de frutos de 12 diferentes aceroleiras (Malpighia emarginata D. C.). Ciência e Tecnologia de Alimentos, Campinas, v. 23, n. 1, p. 101-103, 2003. Disponível em: <http://www.scielo.br/pdf/cta/v23n1/18264.pdf>. Acesso em: 02 maio 2013.

MATIAS, M. F. O.; OLIVEIRA, E. L.; MARGALHÃES, M. M. A.; GERTRUDES, E. Use of fibers obtained from the cashew (Anacardium ocidentale, L) and guava (Psidium guayava) fruits for enrichment of food products. Brazilian Archives of Biology and Technology, Curitiba, v. 48, Special number, p. 143-150, 2005. Disponível em: <http://www.scielo.br/scielo. php?pid=S1516-89132005000400018\&script=sci_arttext $>$. Acesso em: 24 ago. 2015

MELLO, L. M. R. Atuação do Brasil no mercado vitivinícola mundial - panorama 2010. Bento Gonçalves: UVIBRA, 2010. Disponível em: <http://www.cnpuv.embrapa.br/publica/artigos/ mercextvit2010.pdf>. Acesso em: 25 abr. 2013.

MENDEZ, M. H. et al. Tabela de composição de alimentos. Niterói: Universidade Federal Fluminense, 1995.

MONRO, J.; BURLINGAME, B. Carbohydrates and related food compounds: INFOODS tagnames, meanings, and uses. Journal of Food Composition and Analisys, San Diego, v. 9, n. 2, p. 100-118, 1996. http://dx.doi.org/10.1006/jfca.1996.0018.

MOREIRA, A. V. B.; MANCINI-FILHO, J. Influência dos compostos fenólicos de especiarias sobre a lipoperoxidação e o perfil lipídico de tecidos de ratos. Revista de Nutrição, Campinas, v. 17, n. 4, p. 411-424, 2004.

NATIVIDADE, M. Desenvolvimento, caracterização e aplicação tecnológica de farinhas elaboradas com resíduos da produção de suco de uva. 2010. 202 f. Dissertação (Mestrado em Ciência dos Alimentos)-Universidade Federal de Lavras, Lavras, 2010.

NEVES, M. F.; TROMBIN, V. G.; MILAN, P.; LOPES, M. F. F.; KALAKI, R. $O$ retrato da citricultura brasileira. Ribeirão Preto: Markestrat, 2010.

NOGUEIRA, R. J. M. C.; MORAES, J. A. P. V.; BURITY, H. A.; SILVA JUNIOR, J. F. Efeito do estádio de maturação dos frutos nas características físico-químicas de acerola. Pesquisa Agropecuária Brasileira, Brasilia, v. 37, n. 4, p. 463-70, 2002. Disponível em: <http://seer.sct.embrapa.br/index.php/pab/ article/download/6362/3419>. Acesso em: 03 set. 2013.

NÚCLEO DE ESTUDOS E PESQUISAS EM ALIMENTAÇÃO - NEPA. Tabela brasileira de composição de alimentos. Campinas: NEPA-UNICAMP, 2006. 
Qualidade microbiológica e composição de farinhas de resíduos da produção de suco de frutas em diferentes granulometrias STORCK, C. R. et al.

PUUPPONEN-PIMIÄ, R.; NOHYNEK, L.; MEIER, C.; KAÈHKOÈNEN, M.; HEINONEN, M.; HOPIA, A.; OKSMANCALDENTEY, K. M. Antimicrobial properties of phenolic compounds from berries. Journal of Applied Microbiology, v. 90, p. 494-507, 2001. Disponível em: <http://www.ncbi.nlm.nih. gov/pubmed/11309059>. Acesso em: 24 ago. 2015.

ROSS, J. A.; KASUM, C. M. Dietary flavonoids: bioavailability, metabolic effects, and safety. Annual Reviews of Nutrition, Palo Alto, v. 22, p. 19-34, 2002. Disponível em: <http://www.annualreviews.org/doi/pdf/10.1146/annurev. nutr.22.111401.144957>. Acesso em: 03 set. 2013.

SECRETARIA DE ESTADO DA AGRICULTURA E DO ABASTECIMENTO - SEAB. Fruticultura. São Paulo: SEAB, 2015. Disponível em: <http://www.agricultura.pr.gov.br/arquivos/ File/deral/Prognosticos/fruticultura_2014_15.pdf>. Acesso em: 15 set. 2013.
SINGLETON, V. L.; ROSSI, J. A. J. Colorimetry of total phenolics with phosphomolybdic-phosphotungstic acid reagents. American Journal of Enology and Viticulture, California, v. 16, n. 3, p. 144-58, 1965. Disponivel em: <http://garfield.library. upenn.edu/classics1985/A1985AUG6900001.pdf>. Acesso em: 28 jan. 2012.

SOUZA, M. S. B.; VIEIRA, L. M.; SILVA, M. J. M.; LIMA, A. Caracterização nutricional e compostos antioxidantes em resíduos de polpas de frutas tropicais. Ciência Agrotecnológica, Lavras, v. 35, n. 3, p. 554-559, 2011. Disponível em: <http://www.scielo.br/scielo.php?script=sci_ arttext\&pid $=$ S1413-70542011000300017 $>$. Acesso em: 28 abr. 2013.

WANDERZANT, C.; SPLITTSTOESSER, D. F. Compendium of methods for the microbiological examination of food. 3. ed. Washington: American Public Health Association, 1992. 1219 p. 